\title{
TRADUCCIÓN DE LOS TÍTULOS DE LAS PELÍCULAS EN LOS CINES DE ESPAÑA: ¿INGLÉS Y / O ESPAÑOL? ${ }^{1}$
}

\author{
Carmen Isabel Luján García, Universidad de Las Palmas de Gran Canaria \\ Email: clujan@dfm.ulpgc.es
}

\begin{abstract}
This paper has a twofold purpose. On the one hand, it intends to show some of the different strategies used when translating Anglo-American film titles into Spanish. With that purpose, an analysis of the three main linguistic functions (referential, expressive and vocative) has been carried out. The presence of people's and places' proper names in film titles is also considered. On the other hand, this article also includes a sociolinguistic approach of this study by means of the administration of a short survey to a small sample of Spanish cinema viewers of different ages in order to check whether they are able to understand the English titles, among other issues.
\end{abstract}

Keywords: Film titles, translation, English/Spanish, understanding, Spanish audience.

Title in English: Translation of Film Titles in Spanish Cinemas: English and/or Spanish?

Resumen: El presente artículo tiene una doble finalidad, de un lado, pretende mostrar las diferentes estrategias empleadas al traducir los títulos de las películas provenientes básicamente del mundo anglo-americano a la lengua española. Para ello, se han analizado algunas de las principales funciones que se observan en los títulos (referencial, expresiva y apelativa). También se examina la traducción de títulos que comprenden nombres propios de persona o lugar. La segunda parte de este trabajo se centra en una aplicación sociolingüística de este estudio, ya que a través de la administración de una encuesta se indaga sobre si una pequeña muestra de espectadores españoles de distintas generaciones entienden los títulos en inglés, entre otras cuestiones.

Palabras clave: Títulos de películas, traducción, inglés/español, comprensión, público español.

\section{INTRODUCCIÓN}

La traducción de los títulos de las películas que provienen del mundo anglosajón en España ha recibido bastante atención. Distintos autores (JimÉnez SerRano, 1997; DíAz TeiJo, 1997; Navarro Rodríguez, 1997; GonzÁlez Ruiz, 1997) han analizado la traducción de los títulos de películas en el contexto de España atendiendo a diversas realidades y estrategias traductológicas.

Date of reception: 28 May 2010

Date of acceptance: 8 November 2010

Odisea, $n^{\circ} 11$, ISSN 1578-3820, 2010, 301-313 
En el año 2000 Santaemilia llevó a cabo un trabajo en el que analizaba la traducción de los títulos de las películas estrenadas en Valencia desde 1990 hasta 1996 y este autor concluye que la tendencia a la no traducción parece imponerse progresivamente. En sus palabras, "el mercado español asume sin empacho una cantidad impresionante de préstamos (justificados o no)" (SANTAEMILIA, 2000: 210). Como testimonio de la creciente tendencia al alza a la no traducción de los títulos de las películas de origen anglo-americano al español podemos citar otro estudio más reciente llevado a cabo en 2010 (LUJÁN-GARCÍA, 2010), donde se demuestra que cada vez son más los títulos que no se traducen.

Por su parte, Calvo en el año 2000 examinó las diferentes estrategias empleadas a la hora de traducir los títulos de las películas en castellano. Asimismo, también destaca la permeabilidad de la sociedad española hacia lo anglosajón y la omnipresencia del inglés en el mundo publicitario y de mercado.

HeRnÁNDEZ y MENDILUCE en 2005 realizaron un estudio del proceso de traducción de los títulos de las películas y concluyen subrayando la importancia de los factores pragmáticos en este campo. No se debe olvidar que una película es un producto que busca ser vendido a un público, por lo tanto, la función pragmática juega un papel muy importante en este campo.

GonZÁLEz RuIz en 2005 estudió la traducción de títulos fílmicos en el período franquista español. Por su parte, Serrano Fernández (2001) se centró en el análisis de los títulos en el período de transición política español (desde 1975 a 1980).

La traducción de los títulos de las películas, como se refleja en las líneas precedentes, ha sido tratada por diversos autores desde diferentes perspectivas. Sin embargo, todos estos trabajos parecen tener en común el creciente papel dominante de la lengua inglesa en el panorama de los títulos de las películas anglo-americanas en España. Este artículo pretende aportar algo más de luz sobre por qué muchos títulos se mantienen en versión inglesa, mientras que otros se traducen al español. Este hecho nos plantea una serie de cuestiones tanto traductológicas como sociolingüísticas relacionadas con el tipo de estrategia empleada por el traductor al transferir el título.

En primer lugar y, aunque no constituye el objeto de este trabajo, debemos señalar que España es un país con una tradición de doblaje al español en el cine y en la televisión. En la actualidad, aún todas las películas proyectadas tanto en el cine como en la televisión se doblan al español. Sin embargo, también es cierto que ya es posible ver una película extranjera en televisión en versión original a través de la nueva tecnología del TDT. En cuanto a los cines españoles, salvo en eventos especiales, como festivales de cine, es prácticamente imposible ver una película en versión original.

Con respecto a la censura, durante el régimen de Franco, tanto el cine que provenía del extranjero como el que se hacía en España era sometido a un proceso de censura. En este sentido, se podría afirmar que el doblaje de las películas y la traducción de los títulos extranjeros servían al régimen para reforzar y reafirmar el uso del castellano como la lengua nacional (CoLL, 1998:202). Actualmente, no hay proceso de censura, sino más bien existe una calificación de las películas de acuerdo a la edad de los espectadores.

Una vez esbozada brevemente la situación de la traducción en el cine en España, queremos destacar la dificultad que implica la traducción de los títulos de las películas. Se trata de una tarea ardua, ya que dicha traducción no sólo debe responder a una clara necesidad 
lingüística, sino que además ésta debe dar respuesta a una serie de intereses comerciales. Por lo tanto, un título de una película convendría que sintetizara, en la medida de lo posible, el contenido de la misma, con el fin de dar una idea al espectador de qué trata la película que pretende ver. Sin embargo, también es de vital importancia que el título sea atractivo para el espectador, que lo persuada para elegir ese en lugar de cualquier otro. Como bien indica NAVARRo RoDríGUEZ (1997:258), al traducir un título de película "existe una gran concentración de información tanto de tipo lingüístico como de tipo extra-lingüístico y sensorial, información que hay que verter nuevamente en la lengua de llegada".

Considerado el importante grado de dificultad que implica la traducción de títulos de películas, es conveniente tener en cuenta que en el presente trabajo no nos vamos a detener en determinar si los títulos de las películas están traducidos correctamente o no. En nuestro estudio, ofreceremos un estudio actualizado de las tendencias traductológicas en España en cuanto a traducción de largometrajes. Para ello, analizaremos algunas de las distintas estrategias empleadas por los traductores o departamentos comerciales de las distribuidoras, encargados de escoger un título para la película que se va a estrenar en la lengua meta, en este caso, en español. Finalmente, desvelaremos, mediante una pequeña muestra, si una pequeña muestra de espectadores españoles son capaces de entender los títulos de las películas en inglés.

\section{OBJETIVOS}

El presente trabajo tiene una doble finalidad. Por un lado y, en primer lugar, pretende estudiar cuáles son algunas de las funciones empleadas con mayor frecuencia al traducir títulos de películas. En segundo lugar, y con un enfoque más sociolingüístico, este estudio busca desvelar si una pequeña muestra de público español realmente comprende los títulos cuando están en inglés. De ahí que hayamos formulado las siguientes cuestiones como los objetivos de este artículo.

- Analizar cuáles son algunas de las funciones más empleadas en la traducción de títulos de largometrajes del inglés al español, considerando, además los títulos de las películas que incluyen antropónimos o topónimos.

- Averiguar si se busca crear un efecto en el espectador cuando se mantiene el título en inglés y no se traduce al español.

- Por último, se pretende indagar el grado de entendimiento de los títulos por parte de una pequeña muestra de espectadores españoles.

\section{MÉTODO Y MUESTRA}

Con estos objetivos, en la primera parte de este estudio, hemos analizado los títulos de diversas películas estrenadas en España a lo largo de los últimos años, es decir, desde 2006 hasta 2010. Prácticamente todas ellas de origen anglo-americano. Para ello, nos hemos centrado en la cartelera cinematográfica de dos periódicos; uno de tirada nacional, El País, y otro de tirada local, Canarias 7. También nos ha servido como fuente la base de datos de 
películas del Ministerio de Cultura, ya que ésta, además, ofrece la ficha con los datos de las películas calificadas.

Con respecto a la segunda parte de este trabajo, se empleó un cuestionario compuesto de cinco preguntas a una muestra de 40 espectadores, de los cuales 10 pertenecían a cada uno de los cuatro grupos generacionales a los que se preguntó. Dichos grupos abarcaron, por décadas, las edades comprendidas entre 20 y 60 años. Aunque somos conscientes de la limitación de la muestra empleada, por otro lado, consideramos que constituye un primer y pequeño acercamiento a las opiniones de los espectadores españoles de distintas edades.

\section{RESULTADOS}

Dividiremos los resultados en dos secciones distintas; la primera se centrará en el análisis traductológico de los títulos, mientras que la segunda se ocupará de realizar un análisis sociolingüístico.

\subsection{Análisis traductológico de algunas estrategias de traducción empleadas al traducir los títulos de las películas.}

En primer lugar y dada la naturaleza de este estudio consideramos oportuno describir sucintamente cuál es el proceso que se sigue para elegir un título fílmico que atraiga la atención e interés de los espectadores, que es, en definitiva, la principal función de un título, dejando en segundo plano otras funciones tales como las intenciones del autor o director.
El proceso es el siguiente: la agencia de publicidad asociada a la compañía aporta una lista de títulos, que sufre una rigurosa criba en el curso de una reunión en la cumbre ante el presidente de la distribuidora y su departamento de marketing. Tras horas de intensa descarga neuronal, se eligen sólo dos títulos, que son enviados a Los Ángeles para que la productora americana se decida por uno de ellos (Jordi Costa, "Pero ¿quién diablos ha traducido esto?”, el País de las tentaciones, 25.3.1994: 18-19) (en Moya, 2000: 150).

Observamos, pues, que se trata de un proceso donde lejos de primar un criterio académico o lingüístico, lo que impera es la recaudación obtenida. El éxito o fracaso de un film se mide en términos de dólares recaudados en la taquilla.

A continuación, llevaremos a cabo un análisis de distintas estrategias traductológicas empleadas al traducir los títulos de las películas. Es aquí donde el concepto de función pasa a jugar un papel importante, y es conveniente aclarar que a pesar de la confusión o diferentes interpretaciones que existen en torno a este concepto (LvóvSKAYA, 1997:83), en este estudio, nos referiremos a la función lingüística y a la pragmática (o comunicativa) de los títulos de las películas que analizaremos.

Para ello, tendremos en cuenta la tipología establecida por Reib (en CALvo, 2000; 61) en la que se afirma que la traducción de títulos es un translema especialmente complejo, ya que éstos concentran tres de los cuatro tipos de textos: informativo, expresivo y apelativo. 
La función del título fílmico como texto informativo o referencial está más allá de toda duda pues, como comentábamos anteriormente, el título debe aportar al posible espectador una idea del contenido del film al que da nombre. Aquellos títulos que dan prioridad a esta función informativa suelen ser largos y contienen bastante información. A continuación, comentamos algunos casos de títulos que consideramos que se ajustan a este tipo de función traductológica.

Green Zone (2010- Estados Unidos): "Green Zone: distrito protegido"

Wall-E (2007- Estados Unidos): "Wall-E: Batallón de limpieza"

Space Chimps (2008- Estados Unidos): "Space Chimps. Misión espacial”

Wanted (2008- Estados Unidos): "Wanted- Se busca"

The Town (2010- Estados Unidos): "The Town: ciudad de ladrones"

Éstos son ejemplos de títulos que han experimentado una prolongación al ser traducidos del inglés al español. Han mantenido el título original en inglés seguido de una extensión del mismo, lo que también se denomina "adición" o "glosa" en términos traductológicos. Muchas veces las glosas vienen precedidas por doble punto, un punto, una coma, un guión e incluso por paréntesis. Se podría decir que no existe un criterio fijo establecido, como se puede observar en los títulos mencionados donde se emplea dos puntos, un punto o un guión. Con estas adiciones en el título español se pretende aportar una idea del contenido del film al mismo tiempo que se mantiene el título en inglés, lo que puede resultar cool para el espectador español.

Dentro de la función informativa o referencial, también existen títulos que experimentan una modificación total del título original. Esto puede estar motivado porque si se transfirieran en versión original, no dirían nada al público español. Algunos ejemplos son:

\author{
Miss Pettigrew Lives for a Day (2008- Reino Unido): "Un gran día para ellas" \\ Rogue (2007- Estados Unidos): "El territorio de la bestia" \\ Eagle Eye (2008- Estados Unidos): "La conspiración del pánico" \\ Whisper (2007- Estados Unidos): "Hellión, el ángel caído" \\ Mad Money (2007- Estados Unidos): "Tres mujeres y un plan" \\ 3:10 to Yuma (2007- Estados Unidos): "El tren de las 3:10 de James Mangold"
}

Las personas encargadas de traducir estos títulos han considerado que los títulos originales en inglés son insuficientes en términos de función informativa y de ahí que hayan decidido no sólo ampliar el título en el caso de los primeros ejemplos, sino además modificar el contenido del título aportando al espectador cierta información del contenido de la película. Las razones que motivan estas extensiones y/o modificaciones del título pueden ser varias. Por un lado, puede ser que el título original inglés no haya funcionado muy bien en el país de estreno (Estados Unidos, Reino Unido, Irlanda o Australia) y, por eso, se haya cambiado. De esta manera no se predispone negativamente al espectador español. Otra motivación puede ser de carácter cultural, ya que el título en inglés podría no decir nada al espectador español y, por tanto, sea necesario no sólo que se traduzca el título al español sino que, además, se haga ampliando la información que ofrecía el título original. 
Otra de las funciones es la del título como texto expresivo. Ésta se ocupa del empleo de recursos literarios tales como onomatopeyas, neologismos, rima, aliteración, entre otros, con el objetivo de crear un impacto en el espectador. Este tipo de estrategia se emplea con bastante menor frecuencia que la anterior. Algunos ejemplos son:

Donkey Xote (2007- España), es un título donde se hace un juego de palabras con el nombre del famoso personaje de la obra de Miguel de Cervantes.

The Bounty Hunter (2010- Estados Unidos): "Ex-posados". Se hace un juego de palabras con la palabra "esposados" que tiene un doble sentido, el significado de "casados" y el de "arrestados", además del empleo del prefijo "ex" frecuentemente asociado al matrimonio que se acaba.

Biutiful (2010- España). En este título se juega con la palabra inglesa beautiful, cuyo significado es "bello", "bonito", adoptando una ortografía adaptada a su pronunciación original inglesa.

Mystikal (2010- España) es un título donde nuevamente se juega con la palabra inglesa mystical, pero con una alteración en la ortografía original que cambia la $-\mathrm{c}$ por la $-\mathrm{k}$. De nuevo, se pretende impactar al espectador que sepa o no inglés (en español es místico, también con -c ), puesto que se percatará de que la palabra no está escrita "correctamente".

La función del título como texto apelativo es, tal vez, la más frecuente. No debemos olvidar que una película es básicamente un producto que busca ser vendido a una clientela, en este caso, a un público, especialmente cuando se trata de un cine comercial. Esta función contribuye a crear una reacción en el espectador. Algunos ejemplos de este tipo de títulos son:

Atonement (2007- Estados Unidos): "Expiación, más allá de la pasión”. El término inglés atonement significa únicamente expiación. En la versión española se ha añadido la adición o glosa "más allá de la pasión" para atraer a un público que desea ver relaciones pasionales.

The men who stared at goats (2009- Estados Unidos): "Los hombres que miraban fijamente a las cabras" constituye otro ejemplo claro de funcionalidad apelativa, al pretender captar la atención del espectador a través de la palabra "cabra", que no es algo que habitualmente despierte un interés tan grande como para que haya hombres que se dediquen a mirarlas fijamente.

The lovely bones (2009- Estados Unidos): "The Lovely Bones" es un ejemplo más del uso de un adjetivo calificativo como "lovely" o "encantador" para referirse a algo inanimado como son los huesos.

Daybreakers (2009- Estados Unidos): “daybreakers” tiene también una función apelativa al transmitir este título "rompedores de días" una sensación de fuerza y energía al espectador.

En resumen, y vistos los ejemplos anteriores, esta función podría considerarse un tanto más agresiva que las demás en términos de atracción de público.

Dedicaremos también un espacio al análisis de aquellos títulos que emplean nombres propios para denominar un film. Como señala NEWMARK $(1988 ; 214)$ "Normalmente, el nombre propio y los apellidos de la gente se transfieren conservando su nacionalidad y asu- 
miendo que estos nombres no tienen connotaciones en el texto". Asimismo, MoyA (2000:38) aclara que frente a la antigua costumbre de traducir aquellos nombres que contaran con equivalentes en español, "Modernamente, los antroponóminos ya no se suelen naturalizar; simplemente se transfieren, [...] tengan o no correspondencia en nuestra lengua". Por tanto, los títulos con nombres propios tanto de persona como de lugares no suelen traducirse, por el contrario se suelen mantener en su forma original. Algunos ejemplos son:

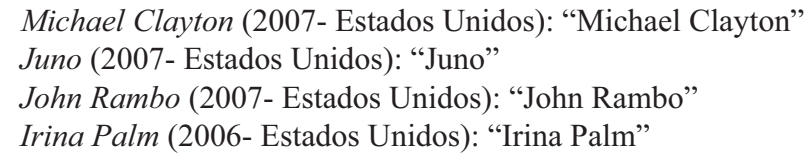

También es posible encontrar ejemplos de nombres propios insertados en un título más largo, en cuyo caso se traduce el resto del título, pero no el antropónimo. Algunos casos son:

Charlie Wilson War (2008- Estados Unidos): "La Guerra de Charlie Wilson"

In the Valley of Elah (2007- Estados Unidos): "En el valle de Elah"

Little Miss Sunshine (2006- Estados Unidos): "Pequeña Miss Sunshine"

Dear John (2010- Estados Unidos): "Querido John"

Percy Jackson \& The Olympians the Lighting Thief (2009- Estados Unidos): "Percy

Jackson y el ladrón del rayo"

Con respecto a los nombres propios de lugares o topónimos extranjeros, como bien indica Moya (2000:45), éstos se suelen transferir al español a no ser que tengan una adaptación o traducción ya arraigada en nuestra lengua. Lógicamente, y como cabría esperar, los nombres propios que suelen aparecer en los títulos de las películas tienden a transferirse. Algunos ejemplos son:

Brideshead Revisited (2008- Reino Unido): "Retorno a Brideshead". Brideshead es un castillo situado en Inglaterra con el que, casi con seguridad, la mayoría del público español no está familiarizado, además de no existir ninguna traducción en español para este nombre, por lo tanto lo más sensato es mantener el nombre original inglés.

Existen también títulos donde se puede observar la combinación de algunas de las funciones, como es el caso del siguiente título:

Sweeney Todd (2007- Estados Unidos): "Sweeney Todd, el barbero diábolico de la calle Fleet"

Aquí observamos la función informativa a través del empleo de la glosa o extensión del título con el fin de orientar al espectador sobre el contenido del film. También vemos la presencia de dos nombres propios, uno de persona y otro de lugar que han sido transferidos sin ningún tipo de traducción. 
La situación también se da a la inversa. Hablamos de aquellos títulos que se estrenan en Estados Unidos en español, tal vez porque confieren un aire de exotismo y acercamiento al contexto de Latinoamérica, que constituye el escenario de estos largometrajes. Asimismo, como señala MoyA (2000:160) "se garantiza la asistencia a las salas de un mercado potencial de 29 millones de hispanoparlantes que viven en Estados Unidos, quienes demandan sobre todo películas habladas en su propia lengua". Tal es el caso de títulos como:

Che, el argentino (2008- Estados Unidos)

Dos colgaos muy fumaos. Fuga de Guantánamo (2008- Estados Unidos)

Bella, la película (2008- Estados Unidos)

No sería justo, asimismo, omitir los casos de otras películas que, a pesar de ser de nacionalidad española, tienen sus títulos íntegros en inglés. Tal vez, con el propósito de parecer más cool o moderno, dada la influencia de la globalización, así como el deseo de intentar llegar a las salas de cine europeas y norteamericanas. Hablamos de títulos como:

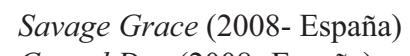

Los resultados revelan que existe una notable presencia de títulos que mantienen su forma original en inglés, frente a un porcentaje considerable que es objeto de traducción al español. Existen una serie de condicionamientos pragmáticos en los criterios empleados a la hora de traducir los títulos. Asimismo, el hecho de que se mantengan muchos títulos en inglés implica también un deseo de parecer moderno o prestigioso, características asociadas al inglés, la lengua del imperio. Por otro lado, las traducciones de los títulos de las películas no sólo son transmisores de un mensaje lingüístico, sino que además, han de crear un impacto en los espectadores.

En cuanto a cuál es la lengua más empleada en los títulos de las películas estrenadas en España en los últimos años, debemos referirnos a dos estudios con carácter cuantitativo que se han llevado a cabo en los últimos años en España. Por un lado, el de SAntaEmilia (2000) en el cual se afirma que un $12,7 \%$ del total de los títulos de películas anglo-americanas estrenadas en España no sufren proceso alguno de traducción, manteniéndose en inglés. Sin embargo, en otro estudio reciente llevado a cabo por LuJÁN GARCía (2010) se sostiene que este porcentaje ha aumentado a un $27,4 \%$ de los títulos que se mantienen en inglés. Estos datos no sólo revelan la creciente tendencia a emplear el inglés en los títulos que se ha experimentado en España a lo largo de los últimos diez años, sino que además refleja que esta última técnica es la más extendida. En este mismo estudio (LuJÁN GARCíA, 2010) observamos que otras técnicas empleadas obtienen porcentajes inferiores. Algunas de ellas son: la traducción literal, con un 18,8\%; la traducción libre con alguna relación con el original (18,8\%); la traducción libre sin ninguna relación con el original $(18,8 \%)$; los títulos que se mantienen en inglés y añaden un subtítulo explicativo e incluso una traducción al español $(9,7 \%)$ y por último, títulos que se traducen al español pero añaden como subtítulo explicativo el título original en inglés $(3,4 \%)$. 
El análisis de algunos títulos que precede estas líneas es muestra del peso que tiene la pragmática en este terreno en particular. Como se ha comentado anteriormente, un título puede aportar una breve idea al espectador del contenido de la película a la que denomina, puede también reflejar una situación o realidad familiar conocida para el espectador, pero como condición indispensable, deberá atraer a un público que pague por ver esa película, ya que será en definitiva esto lo que la convertirá o no en un éxito de taquilla.

\subsection{Análisis sociolingüístico de la incidencia de los títulos de las películas anglo- americanas en la audiencia}

Una vez realizado todo el análisis previo, se nos planteó otra cuestión pragmática sobre si los espectadores realmente entienden los títulos cinematográficos cuando están en versión original inglesa, que, como hemos señalado, parece ser la tendencia que se impone. La duda de si el público de distintas generaciones es capaz de entender el mensaje de los títulos en inglés nos llevó a realizar la última parte de este estudio. Con tal fin, decidimos acercarnos a la puerta de un multicines de Las Palmas de Gran Canaria y preguntar directamente a una pequeña muestra de espectadores de diferentes grupos de edad:

1. comprende entre 20 y 30 años

2. entre 31 y 40 años

3. entre 41 y 50 años

4. entre 51 y 60 años

Se les administró una pequeña encuesta con una serie de cuestiones. En primer lugar, se les preguntó cuál era la principal motivación para elegir una película al ir al cine. Se les ofreció diferentes posibles respuestas (título, comentarios de amigos, trama o sinopsis, otros) y debían elegir una. Los resultados demuestran que el título no es la principal motivación para la elección de una película para los tres grupos generacionales más jóvenes, con unos porcentajes de $20 \%$ ó $0 \%$ (desde los 20 a los 50 años), siendo para éstos los comentarios de amigos y/o familiares, así como la trama del film, más importantes. En cambio, para la generación mayor, entre 51 y 60 años, el título es considerado como la principal razón para elegir una película para un $60 \%$ de los encuestados.

La segunda pregunta es si el título de una película es clave o muy importante para decidirse a ver una película en lugar de otra. Los resultados demuestran que los porcentajes más altos son para la respuesta afirmativa, pues un $80 \%$ de los participantes de los grupos generacionales 2, 3 y 4 responden que el título es importante para la elección de un determinado film. Por el contrario, la generación más joven, el grupo de edad 1, con un $60 \%$, considera que este factor no es determinante a la hora de escoger una película concreta.

La tercera cuestión es si cuando el espectador ve títulos totalmente en inglés es capaz de entenderlos. Las posibles respuestas eran nunca / a veces / siempre y éstas varían en función de los distintos grupos de edad. El grupo 1 presenta un porcentaje mayoritario de un $60 \%$ de informantes que entiende los títulos a veces, frente al restante $40 \%$ que afirma entender siempre los títulos. El grupo 2 presenta un $40 \%$ que entiende los títulos a veces, frente al restante $60 \%$ que escoge siempre. El grupo 3 ofrece una respuesta más diversificada, pues un $20 \%$ nunca entiende los títulos en inglés, mientras que un $60 \%$ los entiende a veces y 
un $20 \%$ que los entiende siempre. Por último, en el grupo 4, una mayoría (80\%) entiende los títulos en inglés a veces, frente a un $20 \%$ que responde que nunca. Podemos determinar, por consiguiente, que la mayoría de respuestas en los cuatro grupos pertenece a la opción que comprende los títulos de las películas en inglés a veces. Existe una familiaridad y un grado de conocimiento del inglés por parte de los espectadores aceptable o suficiente como para comprender los títulos en inglés.

La cuarta pregunta se interesa por si al espectador le gusta ver títulos de películas en inglés, y las respuestas son si / no / me da igual. En el grupo 1, el porcentaje más alto (60\%) es para la opción de que sí le gusta ver títulos en inglés, frente a un $40 \%$ de informantes a los que les da igual. En el grupo 2, las respuestas de sí o no cuentan con los mismos porcentajes (40\%), frente a un $20 \%$ que manifiesta darle igual esta cuestión. El grupo 3 ofrece una posición más crítica, pues las opciones de no o me da igual ver títulos de películas en inglés cuentan con un $40 \%$, frente a un $20 \%$ a los que sí les agrada este hecho. Por último, el grupo 4 también se muestra bastante crítico ya que el porcentaje más alto $(60 \%)$ es de encuestados a quienes no les gusta ver los títulos de las películas en inglés. Deducimos que, cuanto mayor es el espectador, más reacia o negativa es su actitud hacia la presencia de los títulos en lengua inglesa, frente a las generaciones más jóvenes, que muestran una actitud más receptiva.

Por último, la quinta pregunta indaga sobre si los espectadores prefieren las películas norteamericanas sobre las españolas o de cualquier otro lugar. Los encuestados debían elegir entre si / no / no necesariamente y los resultados varían bastante con respecto a la anterior pregunta. En este caso, los grupos 1 y 2 eligen mayoritariamente, con un $60 \%$, la respuesta de que no necesariamente prefieren las películas norteamericanas sobre las españolas o de otros lugares frente a un $40 \%$ que sí prefiere las estadounidenses. Los grupos 3 y 4 expresan, en cambio, preferencia por las películas norteamericanas (con un $60 \%$ el grupo 3 y un $80 \%$ el grupo 4), frente a porcentajes inferiores en las otras dos opciones. De aquí, podemos concluir que las generaciones de más edad expresan una clara preferencia por el cine estadounidense, cosa que no sucede con las generaciones más jóvenes que consideran que no necesariamente estas películas sean las mejores.

Los resultados de la segunda parte de este artículo podrían considerarse limitados, debido a que se centran en una muestra muy pequeña de espectadores de una zona concreta. Sin embargo, conscientes de esta limitación, debemos señalar que se trata de un primer acercamiento a la opinión de los espectadores. Lógicamente, un primer acercamiento que podría verse complementado por un estudio futuro más amplio que se centre en esta cuestión.

\section{CONCLUSIONES}

Tras llevar a cabo un análisis tanto de carácter traductológico como sociolingüístico de algunos títulos cinematográficos, nos encontramos en disposición de emitir las siguientes conclusiones.

Con respecto a las cuestiones planteadas en los objetivos del presente trabajo, hay que señalar que las distintas funciones analizadas en esta investigación pueden observarse a través de los diferentes títulos mencionados. Especialmente la función informativa que, como ya se ha señalado, aporta al espectador una idea de la trama de la película. La función 
expresiva, aunque no con tanta frecuencia, también es empleada como un recurso más, dentro de las posibilidades que ofrece el campo de la traducción de títulos. Por otro lado, el empleo de la función apelativa que, por otra parte, es la más empleada, constituye una estrategia que no sólo tiene una función lingüística, sino además una función pragmática y, por supuesto, comercial.

También se han analizado distintos títulos que constituyen el nombre propio de una persona o lugar, así como otros títulos que contienen nombres propios. En estos casos, hemos visto que, a pesar de que el resto del título se traduce, la tendencia es a mantener los nombres propios, ya sean de personas o de lugares, intactos. Se da una ausencia de traducción en estos casos.

En la siguiente cuestión se plantea si se pretende crear un efecto en el espectador cuando se mantiene el título en inglés, y nuestra respuesta sería que sí. Consideramos que el creciente empleo de la técnica de la transferencia o no traducción de los títulos fílmicos, manteniéndolos en inglés, constituye un reclamo para el espectador. Vivimos en un mundo globalizado y la presencia del inglés en cada ámbito de nuestra vida diaria se ha convertido en algo natural. El cine y los títulos de las películas no son una excepción a esta tendencia.

En lo que se refiere a la última cuestión que se plantea como objetivo en este trabajo, que constituye la segunda parte de esta investigación, los resultados manifiestan que los encuestados más jóvenes parecen ser los que más entienden los títulos de origen angloamericano. La explicación se debe a la mayor exposición y familiaridad de estos informantes con la lengua inglesa, ya que no sólo la han estudiado en primaria, secundaria y estudios superiores, sino que además han estado más expuestos a las influencias de esta cultura. Sin embargo, curiosamente a pesar de que son los informantes más jóvenes los que más entienden los títulos, también son las que más rechazan la idea de que el cine proveniente especialmente de Estados Unidos, sea el mejor. Por el contrario, los participantes más mayores son los que ven este cine como el mejor.

El presente trabajo, con sus limitaciones, ofrece un análisis tanto traductológico como sociolingüístico de la situación actual en el terreno de la traducción de los títulos de las películas estrenadas en España a lo largo de los últimos cuatro años.

En cuanto a las implicaciones de este análisis, cabría señalar que el cine es un área más en la que se pone de relieve la influencia que la cultura anglo-americana está ejerciendo sobre las sociedades occidentales. El hecho de que la mayoría de las películas estrenadas en España provengan de Estados Unidos, la gran industria cinematográfica mundial, junto a la influencia a la que nos acabamos de referir constituyen razones de peso para que nos encontremos no sólo con una infinidad de títulos que se transfieren del inglés a nuestra lengua sin traducción, sino que además, para que el público español casi de cualquier generación esté familiarizado, entienda y acepte de buen grado esos títulos en inglés. Probablemente, esta situación habría sido bien diferente hace unas décadas en España, pues no existía una familiaridad tan grande con la lengua inglesa y la cultura anglo-americana.

Con respecto a futuras vías de investigación, sería de sumo interés llevar a cabo un estudio de caraterísticas similares en otros países europeos con el fin de determinar si los resultados son parecidos a los obtenidos en España. 


\section{REFERENCIAS BIBLIOGRÁFICAS}

CAlvo, J. J. 2000. La cartelera cinematográfica española en versión original ¿síntesis dialéctica de aculturación, o síntoma de sandía afectación?. Ed. F. Fernández. Valencia: Universitat de Valencia. 59-75.

Coll, C.N. 1998. “Language, Film and Nationalism”. Ed. I. GAMBIER. Turku: University of Turku and Centre for Translating and Interpreting. 201-206.

DíAz Teijo, J. T. 1997. La traducción en los títulos de películas del inglés al castellano: Procedimientos y resultados. Eds. Santamaría, J.M. et al. Vitoria: Departamento Filología Inglesa y Alemana. Universidad del País Vasco. 131-141

GonzÁlez RuIz, V. M. 1997. La traducción de los títulos de películas: la importancia del contexto socioeconómico (Una aproximación a su estudio). Ed. RAMírEz JÁIMEZ, A.S. Servicio de Publicaciones Universidad de Las Palmas de Gran Canaria: Las Palmas de Gran Canaria. 125-131.

GonzÁlez Ruiz, V. M. 2001. Ideología y Traducción: estudio de los títulos en castellano de las películas en lengua inglesa estrenadas en España durante el período de la dictadura franquista (1939-1975). Tesis doctoral: Universidad de Las Palmas de Gran Canaria.

GonzÁlez RuIz, V. M. 2001. Ideología y Traducción: estudio de los títulos en castellano de las películas en lengua inglesa estrenadas en España durante el período de la dictadura franquista (1939-1975). Tesis doctoral: Universidad de Las Palmas de Gran Canaria.

Hernández Bartolomé, A. I. y Mendiluce Cabrera, G. 2005. Tradúcelo como puedas: el título de las películas y su traducción inglés/español. Eds. CAmpos PlazA et al.) Granada y Cuenca: Ediciones Atrio y Universidad de Castilla La Mancha. . 559-569

JimÉNEZ SERRANO, O. 1997. El peso de la ausencia: el papel del traductor en la adaptación al español de los títulos de largometrajes en inglés. Eds. Morillas, E. y Arias, J.P. Salamanca: Ediciones Colegio de España. 293-318.

LvósKaya, Z. 1997. Problemas actuales de la traducción. Granada: Granada Lingüística y Método Ediciones.

LuJÁn García, C. I. 2010. "La traducción como herramienta útil en un mundo globalizado. Un análisis de títulos de películas de origen angloamericano traducidos al español". Trickster, vol. 8. Disponible en http://trickster.lettere.unipd.it/doku. php?=lingue_future:garcia_traduccion

Moya, V. (2000). La traducción de los nombres propios. Madrid: Cátedra.

Navarro Rodríguez, A. 1997. Acerca de la traducción de títulos de películas. Eds. SAntamaríA, J.M. et al. Vitoria: Departamento Filología Inglesa y Alemana. Universidad del País Vasco. 257-263. 
Newmark, P. 1988. A Textbook of Translation. Singapore: Prentice Hall.

SAntaemilia, J. 2000. Los títulos de los filmes en lengua inglesa y su traducción al español: ¿Un caos intercultural?. SELL (Studies in English Language and Linguistics), 2: 203-218.

Serrano Fernández, L. 2001. La traducción de los títulos de películas inglés-español en un contexto determinado y determinante: España 1975-1980. Sendebar, 12, pages:153-178.

WEBLIOGRAFÍA:

Internet Movie Database. http://www.imdb.com. (último acceso 30 abril 2010)

Film affinity. http://www.filmaffinity.com (último acceso 30 abril 2010)

MCU (Ministerio de Cultura). Base de datos de películas clasificadas del Ministerio de Cultura del Gobierno Español. http://www.mcu.es/bbddpeliculas/cargarFiltro.do?la yout $=$ bbddpeliculas\&cache $=$ init\&language $=$ es (último acceso 30 abril 2010)

\section{APÉNDICE 1}

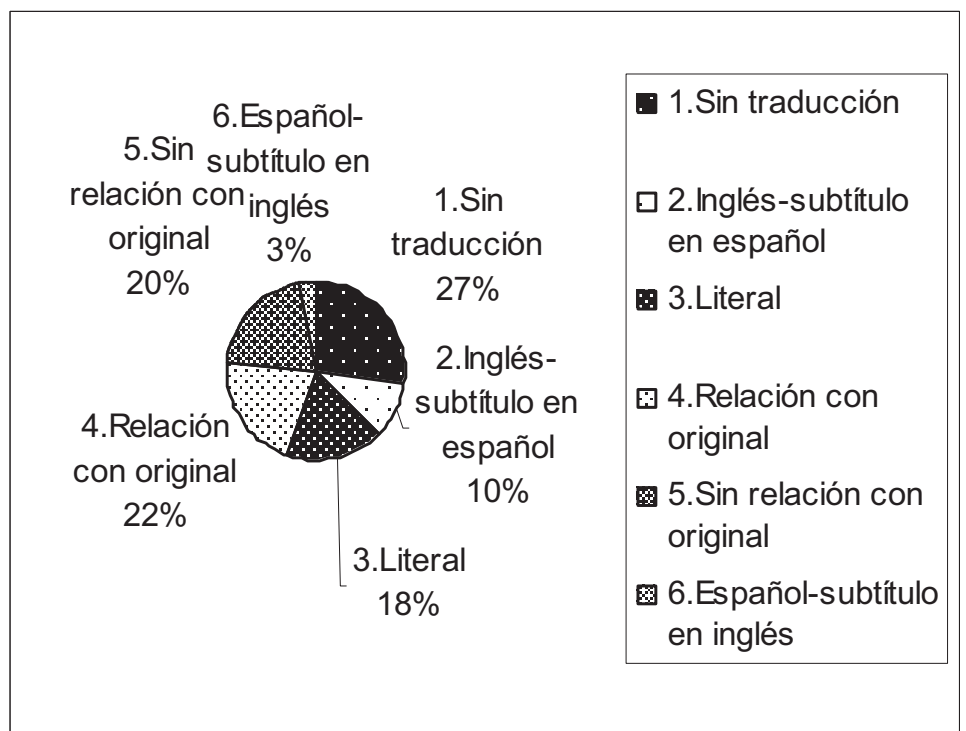

(Sacado de Luján-García, 2010: http://trickster.lettere.unipd.it/doku.php?=lingue future:garcia_traduccion)

Gráfico 1. Técnicas de traducción empleadas en los títulos de películas de inglés a español. 